\title{
Conférence à la mémoire de Helene Hudson Congrès de l'ACIO 2008
}

\section{Patricia Benjaminson}

\section{Abrégé}

Une femme sur neuf développera un cancer du sein au cours de sa vie (Société canadienne du cancer, 2007). Le cancer du sein héréditaire représente seulement de cinq à dix pour cent de tous les cancers du sein; cependant, les femmes porteuses d'une seule mutation génétique de pénétrance élevée ont de quarante à quatre-vingts pour cent de chances de développer un cancer du sein (Nat.Rev. Cancer, 2007). La majorité de ces cancers du sein surviennent chez les femmes âgées de moins de cinquante ans. C'est en 1994 que la mutation génétique BRCAl a été signalée pour la première fois tandis que la mutation génétique BRCA2 l'a été en 1995.

Ce sont souvent les hommes qui sont porteurs de la mutation BRCA2 laquelle est responsable d'approximativement six pour cent des cancers du sein masculins. Les femmes porteuses de cette mutation génétique ont un risque à vie de développer le cancer du sein situé entre cinquante et quatre-vingt-cinq pour cent, un risque de développer un second cancer du sein situé entre trente et cinquante pour cent et un risque de cancer de l'ovaire variant entre dix et vingt pour cent. Chaque parent porteur de la mutation génétique BRCA2 a cinquante pour cent de chances de transmettre cette mutation génétique à ses enfants (NCI, 2006).

Il est difficile de prévoir l'impact émotionnel qu'aura la prise de connaissance d'une telle information sur le risque de cancer. Lorsqu'on fournit à la personne concernée de l'information sur la chirurgie visant à réduire les risques, sur la chimioprévention, sur l'évitement des risques de cancer et sur l'augmentation de la fréquence de dépistage, comment cette personne prend-elle des décisions?

Je vous invite à marcher à mes côtés tandis que je relate comment ma famille a découvert que nous étions porteurs de la mutation génétique fondatrice d'Islande, les pas que nous avons faits ensemble durant le processus de dépistage et enfin, le processus décisionnel suivi par les membres de la famille ayant obtenu un résultat positif au test génétique. Nous allons diriger notre attention sur ma sœur Ritades journées ordinaires, un être extraordinaire.

C'est tout un honneur pour moi d'avoir été choisie pour donner la Conférence à la mémoire de Helene Hudson dans le cadre du congrès 2008 de l'ACIO se déroulant dans la belle ville de Saint John's, TerreNeuve; je suis ravie d'avoir cette opportunité de partager avec mes collègues en soins infirmiers oncologiques une partie de ce que j'ai appris au sujet du cancer du sein héréditaire, du dépistage génétique et des options en matière de réduction des risques.

La Conférence à la mémoire de Helene Hudson vient célébrer le souvenir de Helene Hudson, une infirmière en oncologie exceptionnelle dont nous nous rappelons la vision, la compassion et la détermination. Helene avait un merveilleux sens de l'humour et une vive appréciation du partage du vécu professionnel dans notre discipline. Elle prenait son métier très au sérieux, mais pas sa personne. Helene et l'oncologue de Winnipeg Ian Maxwell ont mis sur pied la Clinique d'oncologie du Victoria General Hospital. Helene était l'une des organisatrices du premier symposium national sur les soins infirmiers en oncologie tenu

Patricia Benjaminson, inf., $C S I O(C)$

Infirmière de recherche, Clinical Investigations Office (CIO)

Action Cancer Manitoba, Winnipeg, Manitoba

pat.benjaminson@cancercare.mb.ca à Winnipeg en 1983 durant lequel se sont tenues les discussions ayant mené à la création de l'ACIO quelques années plus tard. De plus, Helene était un des membres fondateurs de CanSurmount, un groupe de soutien pour les patients atteints du cancer et leurs proches.

En 1989, Helene s'était penchée sur la signification qu'avait pour elle l'exercice des soins infirmiers en oncologie : "Quant à moi, je ne crois pas que l'absence de guérison ou l'évolution de la maladie soient des échecs. La mort n'est pas plus un échec. Nous pouvons tout de même dispenser à ces patients des soins infirmiers marqués par l'intelligence et la créativité, du diagnostic à la guérison ou du diagnostic au décès. ... Il ne faut pas prendre à la légère l'occasion qui nous est donnée d'aller au devant des autres et de les aider de manière significative. C'est là l'essence même des soins infirmiers. Euvrer auprès de patients atteints de cancer nous apporte joie, satisfaction et sens profond. Les patients auprès desquels j'ai travaillé ont touché ma vie de façon exceptionnelle. Les professionnels de la santé sont de vaillants combattants dans la guerre contre le cancer, mais ce sont nos patients qui en sont les véritables héros; leur courage et leur vitalité nous sont d'une grande inspiration. »

Avant son soudain décès en 1993, Helene a touché la vie de bien des individus qui se souviendront à jamais de son dévouement envers les patients atteints du cancer et envers leurs proches. Le rire de Helene résonne encore dans la mémoire des nombreuses personnes qu'elle a soignées ou avec qui elle a travaillé.

Dans ma conférence, je fais un survol rapide des mutations génétiques associées au gène $\mathrm{BRCA}$, je présente un bref historique du cancer du sein dans ma famille et je discute du dépistage génétique et des options en matière de réduction des risques à la fois pour les hommes et pour les femmes ayant obtenu un résultat positif au test de mutation génétique BRCA. Je me concentre sur la prise de décision et sur les opérations chirurgicales de ma sœur Rita, sur l'annonce de la nouvelle aux enfants, un vrai dilemme, et sur la vie que l'on mène quand on sait qu'on court un risque élevé de développer un cancer du sein ou d'autres types de cancers.

\section{Les mutations génétiques BRCA}

On estime que de 5 à 10 pour cent de tous les cancers sont attribuables à des mutations héréditaires de gènes particuliers participant à la régulation associée au cancer. Un gène suppresseur de tumeurs ayant muté ou défectueux ne pourra pas empêcher la prolifération incontrôlée des cellules, un processus qui rend la cellule incapable de supprimer les changements aboutissant au cancer.

On pense que les gènes BRCA1 et BRCA2 sont des gènes suppresseurs de tumeurs qui jouent un rôle au niveau de la réparation de l'ADN, de la transcription génétique et du contrôle du cycle cellulaire. Le gène BRCA1, découvert en 1994, se trouve sur le chromosome 17. Quant au gène BRCA2 dont la découverte remonte à 1995, il est situé sur le chromosome 13. Les tests commerciaux permettant de détecter les mutations au niveau de ces gènes existent depuis 1996. Près de 2000 mutations et variations de séquence distinctes ont été décrites pour les gènes BRCA1 et BRCA2.

Quoique de nombreux facteurs influencent la probabilité de développer un cancer du sein, une mutation héréditaire au niveau du gène BRCA1 ou BRCA2 est le plus fort prédicteur véridique du cancer du sein connu à ce jour.

En Amérique du Nord, il est estimé qu'une personne sur 300 à 800 est porteuse de mutations génétiques BRCA. Beaucoup de gens supposent, à tort, que la transmission paternelle est impossible; cependant, la transmission des mutations au niveau de ces gènes est 
une transmission autosomique dominante. Cela signifie qu'un gène muté peut être transmis à la descendance par l'un ou l'autre des parents. Un parent porteur de la mutation génétique a $50 \%$ de chances de transmettre le gène modifié à chacun de ses enfants. En d'autres mots, chaque enfant d'un parent porteur à une chance sur deux d'hériter la mutation génétique. En outre, il suffit d'hériter d'une seule copie du gène modifié pour courir un risque accru.

Un petit nombre de mutations courantes, appelées mutations fondatrices, ont été identifiées au sein de groupes ethniques particuliers. Par exemple, deux mutations BRCA1 spécifiques et une mutation BRCA2 spécifique sont fréquentes chez les Juifs ashkénazes ayant des racines en Europe centrale et orientale.

La mutation fondatrice islandaise « BRCA2 999 del 5 » est la seule mutation fondatrice islandaise de grande fréquence parmi la population d'origine islandaise.

Chez les femmes porteuses d'une mutation génétique BRCA2, le risque à vie de développer un cancer du sein peut atteindre $85 \%$, $50 \%$ pour ce qui est d'un second cancer du sein et $20 \%$ pour ce qui est d'un cancer de l'ovaire. Chez les hommes, le risque de développer un cancer du sein dépasse $6 \%$, et ils courent un risque accru de développer le cancer de la prostate qui, comme certaines études l'ont indiqué, est plus agressif que dans les cas sporadiques. De plus, l'on pense que les hommes et les femmes courent un risque accru de développer les cancers suivants : pancréas, cavité buccale, pharynx, estomac, vésicule biliaire, mélanomes et cancers hématopoïétiques.

\section{Survol des antécédents familiaux}

Mon grand-père Skuli Benjaminson est né dans un petit village d'Islande en 1879. Il a immigré au Canada en 1883 en compagnie de ses parents et de sa sœur aînée. Ils se sont établis sur le littoral du lac Winnipeg à 16 kilomètres au nord de Gimli, Manitoba. En 1895, lorsque Skuli avait 16 ans, son père est décédé après plus de deux années de mauvaise santé.

Quelques années plus tard, Skuli a épousé Laufey, elle aussi de descendance islandaise et, en l'espace de 6 ans, ils ont eu quatre 4 enfants, deux filles et deux garçons. Laufey est morte en 1921 à la suite d'une maladie prolongée alors que leur plus jeune enfant, mon père Fred, n'avait que deux ans. Nous ne connaissions pas la cause de son décès.
Lorsque Setta, la deuxième femme de mon grand-père, a subi un accident cérébrovasculaire débilitant, la sœur célibataire de mon père, ma tante Inga, est retournée à la maison de ses parents pour aider à la soigner. Les membres de notre famille élargie étaient très proches les uns des autres et se réunissaient fréquemment pour le souper du dimanche dans la maison que grand-père avait bâtie à la périphérie de Winnipeg.

En 1965, pour pouvoir faire face aux besoins de santé de plus en plus lourds de Setta, mes grands-parents, tante Inga, mes parents, mes deux frères, mes deux sœurs et moi avons tous aménagés dans une maison à huit chambres afin que ma mère, elle-même une infirmière, puisse s'occuper de Setta.

Tante Inga a été diagnostiquée d'un cancer du sein à l'âge de 46 ans. Elle a été admise à l'hôpital le 16 février 1963 pour y subir une mastectomie radicale et a reçu son congé trois semaines plus tard, le 7 mars. Inga a commencé sa radiothérapie le 4 avril.

Étant donné que nous vivions tous dans la même maison, nous avons partagé avec tante Inga certaines expériences que la plupart des enfants ne font pas habituellement. Inga souffrait d'un énorme lymphœdème au bras droit dont la force était très réduite. Elle éprouvait également des épisodes dépressifs récidivants et avait du mal à s'adapter à l'altération de son image corporelle et à l'évolution de ses rôles personnels et professionnels. Une inscription dans le journal familial en date du 12 avril indique qu' "Inga est d'humeur très maussade aujourd'hui ». Elle exprimait régulièrement la gêne qu'elle ressentait lorsque sa prothèse se libérait de son soutien-gorge et apparaissait au-dessus de l'encolure de son corsage ou de sa robe ou encore lorsqu'elle avait de la difficulté à se relever d'une chaise.

Tante Inga est morte en février 1990 d'un carcinome métastatique d'origine inconnue.

En janvier 1992, alors qu'elle était en vacances en ColombieBritannique avec son mari, ma sœur Rita, alors âgée de 44 ans, s'est aperçue qu'elle avait une masse dans le sein gauche. Elle savait, intuitivement, qu'elle avait le cancer du sein et dès son retour au Manitoba, a fait tout ce qu'il fallait pour obtenir un rendez-vous avec un chirurgien. Je l'ai accompagnée au cabinet du chirurgien à des fins de diagnostic. La biopsie qu'elle a subie a révélé la présence d'un comédocarcinome intracanalaire infiltrant de grade 3, T1 N0 M0 avec récepteurs d'estrogène et de progestérone négatifs. Rita a fait face à son

BRCA1
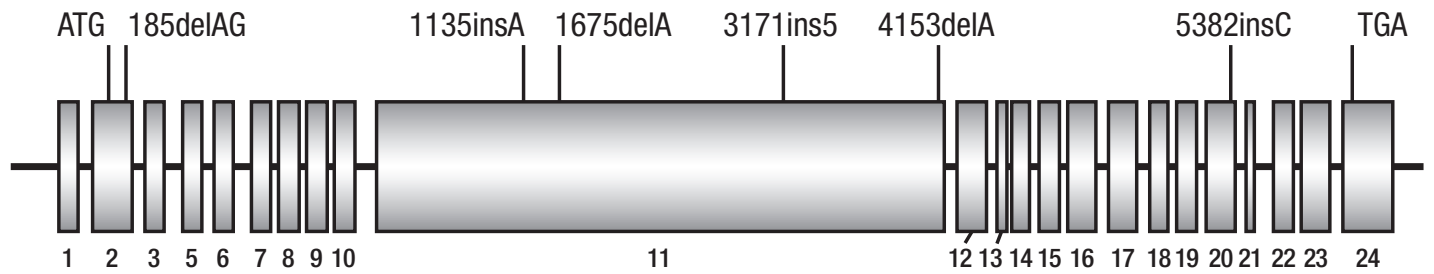

BRCA2
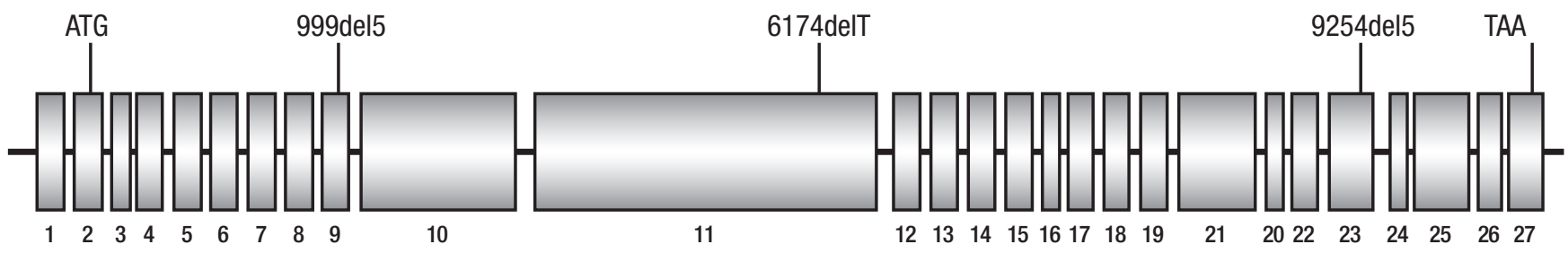

Source: Nat Rev Cancer @ 2007 Nature Publishing Group

Figure 1. 
diagnostic et à son traitement avec une détermination incroyable, avec humour et avec une grande confiance dans l'avenir. Elle a subi une exérèse tumorectomie et une dissection axillaire suivies d'une radiothérapie. Par la suite, Rita a reçu un traitement de chimiothérapie dans le cadre de l'essai clinique NSABP B.23. Il s'agissait d'un essai clinique mené à double insu avec répartition aléatoire des femmes recevant un protocole $\mathrm{CMF}$ ou un protocole $\mathrm{AC}$ avec ou sans tamoxifène. Rita a reçu ses traitements de chimiothérapie au service d'oncologie de l'Hôpital général Victoria à Winnipeg. L'une des merveilleuses infirmières en oncologie administrant les traitements et prodiguant les soins à Rita n'était nulle autre que Helene Hudson. Rita est une technologue de laboratoire et une technicienne en radiologie qui prend toujours le temps de fournir de l'information, de se soucier du soin et du confort des patients et de leurs proches et de placer les interactions avec les patients sous le signe d'un humour enjoué; eh bien, les soins qu'Helene a prodigués à Rita étaient caractérisés par ce même type d'humour et par ce même sentiment de compassion.

En février 2006, notre nièce de 40 ans a découvert qu'elle avait une masse au sein. Sa biopsie au trocart s'est révélée positive et, tandis qu'elle attendait sa chirurgie, elle s'est mise à faire des recherches sur le diagnostic et sur le traitement du cancer du sein. La province où elle habite connaît actuellement une pénurie aiguë d'oncologues médicaux. Non contente de devoir attendre passivement, elle a fait les arrangements nécessaires pour subir une tomographie TEP en dehors de sa province, laquelle a révélé la présence d'un ganglion axillaire animé d'une activité métabolique. Elle a alors subi une mastectomie ainsi qu'un évidement ganglionnaire avec reconstruction immédiate du sein puis a entamé la très longue attente, aux dires des intervenants de la santé, pour voir un oncologue médical.

Dans un de ses messages électroniques, elle écrivait « Tante Pat - je ne sais pas comment je vais composer avec ce contretemps - j'ai l'impression d'avoir dans le corps une bombe à retardement sur le point d'exploser ». Avec sa permission, j'ai commencé à préconiser activement son premier traitement et je l'ai aidé à naviguer dans le dédale du système de soins de santé. Lors d'une discussion avec l'une des infirmières référantes de son centre de cancérologie local, nous avons fait les arrangements pour une consultation au Centre de cancérologie du Manitoba à Winnipeg. Les investigations techniques ont été réalisées, et elle a pris des rendez-vous pour une évaluation en radio-oncologie et une autre en oncologie médicale, puis a reçu son premier cycle de chimiothérapie à Winnipeg. Ceci fait, sa province de résidence pouvait prendre le relais pour ses soins et elle a pu poursuivre ses traitements sans contretemps. Elle se faisait accompagner, à ses rendez-vous, soit par sa tante Rita soit par moi-même, et ce, à sa demande. Le radio-oncologue a passé en revue nos antécédents familiaux et nous a référées à la clinique du cancer du sein héréditaire.

\section{Le dépistage génétique (Comment nous avons découvert le problème)}

Il est important que toutes les femmes jugées à risque d'être porteuses d'une mutation génétique BRCA soient dirigées vers une clinique spécialisée en cancer du sein héréditaire pour y recevoir des consultations génétiques et la prise en charge qui s'impose.

L'information génétique est complexe et il arrive souvent qu'elle soit mal interprétée par les personnes possédant peu de connaissances médicales. Au Canada, la couverture offerte dans le cadre de l'assurance maladie n'est pas modifiée par les résultats du dépistage génétique, mais il est possible que ceux-ci soient utilisés par les compagnies d'assurance sur la vie pour refuser d'offrir une couverture aux individus réputés porteurs d'une mutation génétique ou d'en limiter la portée. Comme il est obligatoire de divulguer ces renseignements si on vous le demande, il est conseillé de passer en revue toutes vos polices d'assurance - notamment les polices d'assurance vie et d'assurance invalidité - avant de subir des tests génétiques. Aux États-Unis, une nouvelle loi fédérale «Genetic
Information and Nondiscrimination Act » a été adoptée en mai 2008; lorsqu'elle entrera en vigueur, elle fournira une protection à cet effet en matière d'assurance et d'emploi.

Le dépistage génétique commence toujours auprès d'un membre d'une famille qui vient d'être diagnostiqué d'un cancer du sein ou de l'ovaire. Toutefois, les résultats du dépistage génétique ne touchent pas uniquement la personne ayant subi les tests mais l'ensemble de la famille biologique de cette personne. Il y a de nombreuses questions qu'il convient d'examiner avant de se livrer au dépistage génétique. Y a-t-il un moment idéal pour engager la participation de la famille? Est-ce avant le dépistage? Ou bien une fois que les résultats ont été obtenus? Et si je ne veux pas partager mes résultats? Et si certains membres de la famille ne veulent rien savoir? Ceci peut-il avoir une incidence sur mes assurances? Ces connaissances auront-elles un impact sur l'avenir de mes enfants? Est-il possible qu'une personne ne veuille pas épouser un individu porteur d'une mutation génétique? Ou encore employer un tel individu?

La conseillère en génétique examine l'information médicale familiale concernant notamment les parents du premier degré (parents, frères et/ou sœurs, enfants), ceux du deuxième degré (tantes, oncles, grands-parents) et ceux du troisième degré (cousins, cousines). Cela peut être ardu car il est possible que nous ignorions l'information médicale de nos proches. La conseillère en génétique doit posséder un ensemble de connaissances et avoir la capacité et la sensibilité requises pour fournir du soutien aux patients et à leur famille du début à la fin du processus. Afin que les patients puissent donner leur consentement éclairé, elle doit dispenser un enseignement et des conseils, notamment en ce qui concerne le but du dépistage génétique, les répercussions des résultats éventuels des tests (y compris les effets psychosociaux), les implications pour la famille, quand, comment et par qui les résultats seront communiqués et les options relatives à la réduction des risques ou à la surveillance accrue.

La Clinique du cancer du sein héréditaire d'Action Cancer Manitoba encourage les patients à se faire accompagner de membres de la famille lors de leurs rendez-vous, s'ils le souhaitent. Notre nièce a ainsi invité sa sœur, son père (mon frère), Rita et moi. Le rôle que je m'étais donné pour ces rendez-vous était de prendre des notes, de poser les questions qui convenaient et d'être le porte-parole et le soutien de ma famille.

Connaître ses propres caractéristiques génétiques est potentiellement habilitant, effrayant ou même accablant. Il est important de réfléchir à ce que vous allez éprouver et ce, avant de vous livrer au test. Comme l'une de mes collègues infirmières me l'a confié en parlant d'une de ses amies «C'est bizarre comme il y a des gens qui préfèrent ne pas savoir et s'en sentent d'autant mieux ».

Le dépistage génétique s'accompagne de risques psychologiques et psychosociaux. Les individus qui se savent porteurs d'une mutation susceptible de provoquer le cancer peuvent ressentir anxiété, dépression, colère et éprouver des sentiments de vulnérabilité et de culpabilité d'avoir possiblement transmis la mutation à leurs enfants.

La conseillère en génétique doit faire une évaluation attentive de la réaction de l'individu à l'annonce de la nouvelle. Au cours de notre première visite, malgré qu'on lui avait rappelé que Rita était prémonopausée au moment du diagnostic, le médecin résident se montrait inflexible et insistait, au départ, que notre nièce était la seule personne qui soit admissible au dépistage initial. Lorsque le médecin résident a quitté la pièce, notre nièce a déclaré qu'elle ne souhaitait pas partager ses résultats avec le reste de la famille puisqu'elle ne voulait pas que ses enfants prennent connaissance des résultats et que si elle ne les communiquait à personne d'autre, il n'y avait aucun risque qu'ils l'apprennent par inadvertance. Vous comprendrez que notre propre réaction initiale à cette déclaration était plutôt émotionnelle et qu'elle était susceptible d'augmenter fortement le stress au sein de la famille. Sa sœur, leur tante Rita et moi avons toutes trois eu la même réaction« et nos enfants, alors! » Heureusement, nous avons toutes été capables de garder notre calme et l'avons appuyée dans sa démarche mais j'ai réalisée avec quelle facilité des situations de ce genre pourraient causer 
une détresse considérable au sein d'une famille. La conseillère en génétique nous a assuré avec douceur que les résultats de dépistage individuels resteraient confidentiels mais que le dépistage serait offert aux membres appropriés de la famille en vertu de ces résultats. Quand le généticien est revenu avec le médecin résident, il a accepté que le dépistage initial concerne à la fois Rita et notre nièce. Du fait de notre origine islandaise, le test génétique porterait avant tout sur des allèles (gènes allélomorphes) liés à ce groupe ethnique particulier.

Le dépistage génétique de familles peut exiger une très longue période de temps. Les patientes ayant eu le cancer du sein ou de l'ovaire subissent le test dans un premier temps et si leurs résultats sont positifs, c'est au tour de leurs frères et sœurs et de leurs enfants (s'ils sont d'âge adulte). Il faut compter jusqu'à trois mois pour obtenir les résultats individuels. La prise de contact avec les membres de la famille élargie peut s'avérer complexe, surtout dans une famille d'origine islandaise où les noms pullulent au sein d'une même famille et où l'on n'est pas toujours certain de savoir qui est cousin biologique de qui.

Il y a, fondamentalement, trois résultats éventuels :

- un résultat positif signifie qu'une mutation génétique a été découverte;

- un résultat négatif par rapport à une mutation démontrée dans la famille (que l'on appelle également « vrai négatif »);

- un résultat négatif sans que l'on sache s'il y a une mutation familiale particulière (considéré « indéterminé » ou « non concluant »-dans ce cas, on n'aura obtenu pour ainsi dire aucune nouvelle information).

En règle générale, on pense qu'un résultat vrai négatif signifie que la personne court les mêmes risques que la population en général, quoique cette affirmation soit entourée d'une certaine controverse. Dans la population générale, le risque à vie de développer un cancer du sein s'élève à $11-14 \%$.

La figure 2 illustre les cas de cancer dans notre arbre généalogique. Le gris y représente les cas de cancer du sein et le noir les autres cancers non reliés aux mutations génétiques BRCA. Les deux cases du haut représentent mes grands-parents paternels-j'ai mis un point d'interrogation sur chacun d'eux puisque nous ne savons pas lequel des deux était porteur de la mutation génétique. Parmi leurs quatre enfants, nous savons que mon père (la case la plus à droite portant le symbole «+ ») était un porteur. Tante Inga l'était probablement elle aussi et il se peut que mon oncle était également porteur de la mutation génétique étant donné que deux de ses trois filles ont eu le cancer du sein. Les résultats de leurs tests ne sont pas encore connus. J'ai ajouté les générations venant après la mienne uniquement pour les porteurs de la mutation. Parmi mes frères et sœurs et moi-même, trois des cinq

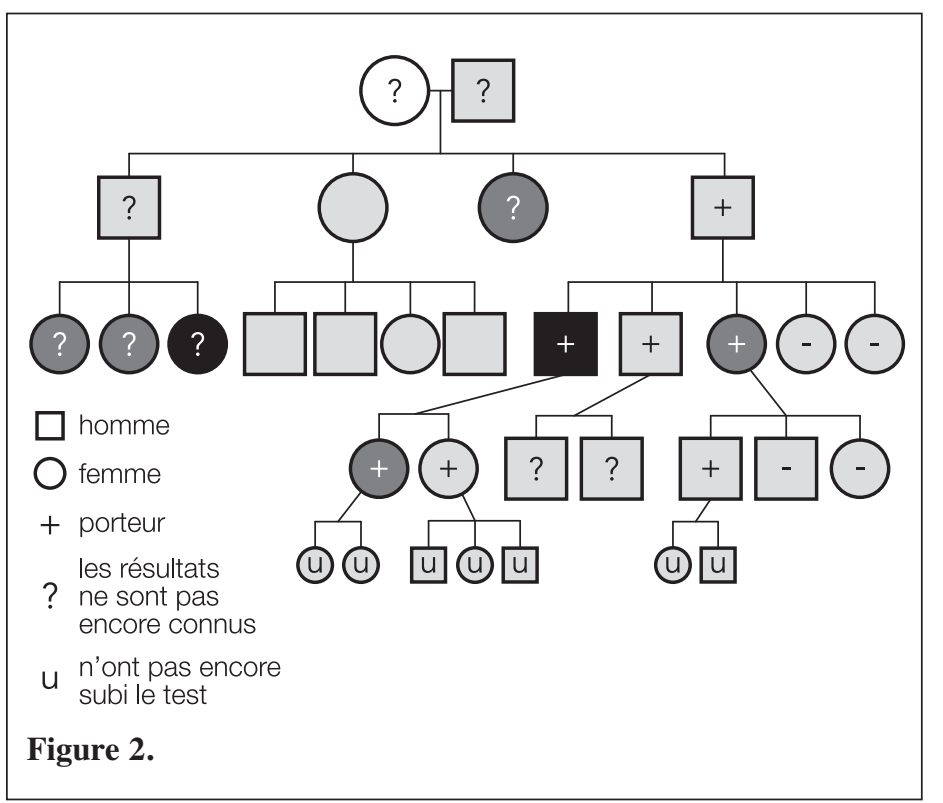

ont obtenu un résultat positif et parmi nos enfants, trois sont positifs, 2 négatifs et 2 n'ont pas encore subi le test. Leurs enfants à eux n'ont pas encore subi le test puisqu'ils sont tous encore mineurs.

Les cancers qui frappent les individus porteurs de la mutation génétique BRCA1 ou BRCA2 ne se produisent pas durant l'enfance. Comme la dispensation du dépistage génétique aux mineurs ne présente aucun avantage médical dans le cas présent, il est conseillé d'attendre que ces individus aient atteint l'âge de la majorité et qu'ils prennent une décision éclairée sur le dépistage génétique. Quand ces enfants-là atteindront l'âge adulte, on peut s'attendre à ce que l'on dispose de stratégies plus efficaces de surveillance et de réduction des risques.

\section{Les sentiments des "épargnés "}

L'impact émotionnel de la prise de connaissance des résultats des tests génétiques varie d'un individu à l'autre. Une personne ayant déjà eu un diagnostic de cancer peut avoir une réaction très différente de celle d'une autre personne ayant obtenu un résultat positif mais n'ayant jamais eu le cancer auparavant. Les individus qui apprennent qu'ils ne sont pas porteurs d'une mutation peuvent éprouver un mélange de soulagement et de culpabilité extrêmes. Dans notre famille, il était facile de partager les résultats négatifs aux tests avec Rita puisqu'elle avait laissé savoir qu'elle priait pour que tous les résultats soient négatifs et qu'elle ne ressentait aucun des sentiments du genre «pourquoi moi et pas toi » se manifestant fréquemment dans d'autres familles.

Par contre, en ce qui concerne les personnes qui s'étaient préparées à l'annonce d'un résultat positif au test, on rapporte qu'elles avaient de la difficulté, à l'annonce de leur résultat négatif, à " retourner », émotionnellement parlant, au niveau de risque associé à la population en général. Elles continuaient de s'estimer à risque sans pour autant bénéficier des options permettant de faire baisser le risque perçu. $\mathrm{Ce}$ sentiment est encore rehaussé par la controverse régnant dans la littérature puisque certaines études semblent indiquer que les individus dont les résultats sont négatifs courent malgré tout un risque accru.

Étant donné que les membres de chaque génération d'Islandais changent leur nom de famille et que les hommes et les femmes ont des noms de famille légèrement différents, je plaisantais souvent avec mon père, quand j'étais petite, que chaque personne d'origine islandaise que je rencontrais devait être un de mes cousins ou une de mes cousines. Pendant mon enfance, j'étais fière de mes origines islandaises et de mes «excellents gènes islandais ». Lorsque j'ai découvert que ma famille était porteuse d'une mutation génétique fondatrice, j'ai ressenti un écrasant sentiment de colère envers la trahison commise par nos ancêtres.

\section{Réduction des risques/surveillance : hommes}

Les hommes qui héritent d'une mutation génétique BRCA courent un risque accru de développer le cancer, mais la hausse en est bien moins spectaculaire que chez les femmes. Selon certains rapports, malgré ce risque accru de cancer et peut-être parce que la différence n'est pas aussi spectaculaire que chez les femmes, il semblerait que la priorité soit accordée à l'information et au dépistage génétique des femmes dans les familles concernées. Dans notre cas, les hommes de la famille ont reçu une attention semblable à celles des femmes lors de la séance d'information et du processus de dépistage, mais les hommes ayant obtenu un résultat positif n'ont reçu aucune information - ou très peu-relativement à la réduction des risques / à la surveillance accrue et n'ont pas fait l'objet de références ultérieures. Avec leur permission, leurs médecins de famille respectifs ont été contactés mais c'est à eux seuls que revenait le fardeau de pousser plus loin les choses. J'ai donc remis à chacun de mes frères et de mes neveux un exemplaire des Recommandations de gestion du HBOC [cancer héréditaire du sein et/ou de l'ovaire] du NCCN destinées aux hommes dont voici l'essentiel : 
- une formation en matière d'auto-examen des seins et la pratique mensuelle de ce dernier;

- un examen clinique des seins à tous les six mois;

- la possibilité de faire effectuer une mammographie de référence, une mammographie annuelle si l'examen de référence révèle une gynécomastie ou une densité parenchymateuse/glandulaire au sein;

- l'adhésion aux lignes directrices de dépistage du cancer de la prostate décrites dans les lignes directrices de détection précoce du cancer de la prostate du NCCN.

\section{Réduction des risques/surveillance : femmes}

À l'opposé des cas sporadiques de cancer du sein, les cas mettant en jeu le BRCA ont tendance à avoir une pathologie médullaire; des récepteurs d'estrogènes et de progestérone négatifs; un grade plus élevé. Lorsqu'elles sont comparées aux femmes atteintes d'un cancer du sein sporadique après 5,10 et 15 ans, les femmes porteuses du BRCA ayant subi une tumorectomie et une radiothérapie courent un risque considérablement plus élevé d'une récidive locale et d'un cancer du sein controlatéral.

Les données semblent indiquer que certains individus porteurs de mutations de BRCA ne développeront jamais de cancer du sein ou de l'ovaire au cours de leur vie; malheureusement, l'identification de ces individus est actuellement impossible.

Comme avec toutes les statistiques que nous utilisons auprès de nos patients lorsque nous discutons du risque et des résultats pour le patient, le risque n'est pas le risque qui s'applique à un patient particulier, mais plutôt le risque moyen pour l'ensemble des patients. Nous ne pouvons donc pas avancer «Rita, vous avez une probabilité de $60 \%$ d'être atteinte d'un autre cancer du sein » étant donné que nous ne possédons pas les connaissances qui nous permettraient d'être si précis-nous devons plutôt lui dire qu'elle court un risque pouvant atteindre un certain chiffre alors qu'en réalité, il est possible qu'elle ne développe jamais un autre cancer. Il importe de souligner le fait que les individus ayant obtenu un résultat positif au test ne développent pas nécessairement tous le cancer. De même, ce ne sont pas toutes les personnes qui ont obtenu un résultat positif au test et ont déjà eu un cancer du sein qui contracteront un nouveau cancer du sein. C'est la raison pour laquelle les décisions liées à la réduction des risques sont si complexes. Le rôle de la conseillère en génétique et des infirmières en oncologie est d'une importance cruciale si l'on veut aider les patients à comprendre que la seule bonne réponse est la réponse qu'ils choisissent.

Certains chercheurs croient que le risque de cancer du sein décline avec l'âge chez les porteurs de mutations génétiques BRCA1 mais pas chez les porteurs de mutations génétiques BRCA2.

\section{Lignes directrices de pratique en oncologie $\mathrm{HBOC}$ [cancer héréditaire du sein et/ou de l'ovaire] du \\ NCCN, 2008, recommandations pour les femmes :}

- formation à l'auto-examen des seins (AES) et AES mensuel à partir de 18 ans;

- examen clinique des seins à partir de 25 ans;

- mammographie et IRM des seins de dépistage annuelles à partir de 25 ans (individualisés en fonction de l'apparition la plus précoce dans la famille);

- discuter de la mastectomie en tant qu'option de réduction des risques;

- discuter de la salpingo-ovariectomie en tant qu'option de réduction des risques;

- pour celles qui choisissent de ne pas subir la chirurgie de réduction des risques, une ultrasonographie transvaginale concurrente + CA-125 tous les 6 mois à 35 ans ou bien 5-10 ans avant l'apparition la plus précoce du premier diagnostic de cancer de l'ovaire dans la famille;

- envisager la chimioprévention;

- envisager de participer aux études expérimentales d'imagerie et de dépistage, lorsqu'elles sont disponibles
Il importe de réitérer à maintes reprises que les femmes ayant obtenu un résultat positif à une mutation génétique de BRCA doivent faire face à des choix difficiles en matière de gestion des risques de cancer. D'ailleurs, peu de données sont disponibles sur le processus décisionnel.

Afin de simplifier légèrement les choses, les infirmières peuvent aider les patients à comprendre qu'il y a trois options fondamentales : la surveillance accrue, la chimioprévention, la chirurgie prophylactique. Il convient d'expliquer attentivement ces options.

(Quelques auteurs, de moins en moins nombreux, envisagent encore une quatrième option :

- Évitement des risques de cancer

Certains comportements de vie comme un exercice régulier et la réduction de la consommation d'alcool, diminuent le risque de cancer; bien qu'il a été démontré qu'ils réduisent le risque dans la population en général, les effets sur les individus porteurs des mutations génétiques de $\mathrm{BRCA} 1$ ou $\mathrm{BRCA} 2$ demeurent inconnus à ce jour.)

Aucune de ces options n'élimine le risque de cancer.

\section{Surveillance}

Une surveillance attentive permettra de détecter un nombre important, mais pas la totalité, des cancers du sein précoces. Les porteurs d'une mutation du gène BRCA doivent apprendre l'autoexamen des seins et le pratiquer sur une base mensuelle. Dès l'âge de 25 ans, il leur faut subir une IRM et une mammographie annuelles ainsi que des examens cliniques des seins à tous les six mois.

Le dépistage du cancer de l'ovaire tel que précisé dans les lignes directrices du NCCN est considéré par beaucoup comme étant inefficace et n'est donc pas une option offerte dans de nombreux centres. Un nouvel examen sanguin capable, espère-t-on, de détecter le cancer de l'ovaire avec une exactitude de $99 \%$ subit actuellement une évaluation de phase 3 .

Il est important de souligner auprès des patients que le meilleur résultat d'une approche de surveillance est de détecter le cancer du sein ou de l'ovaire au stade précoce. Il est évident que cette approche ne permet pas d'éviter le cancer.

\section{Chimioprévention}

La chimioprévention est une autre option. Il a été démontré que le tamoxifène réduit le risque de cancer du sein d'environ $50 \%$ chez les porteurs d'une mutation BRCA2 mais pas chez ceux d'une mutation BRCA1.

\section{Chirurgie prophylactique}

Quoique certains observateurs voient en elle des interventions par trop radicales et controversées, la chirurgie prophylactique constitue la plus efficace de toutes les stratégies de prévention disponibles. Selon les estimations, la salpingo-ovariectomie bilatérale de nature prophylactique réduit le risque de cancer de l'ovaire de 85-95 \% et celui de cancer du sein de 46-68 \%. Quant à la mastectomie prophylactique bilatérale, elle réduit le risque de cancer du sein de 85 $95 \%$. Ces deux types d'opération s'accompagnent de risques et d'avantages potentiels qui viennent compliquer la prise de décision.

Quel est le moment propice pour subir une chirurgie prophylactique?

\section{La prise de décision (Rita)}

Lorsqu'elles ont reçu des résultats positifs au test génétique, les femmes de notre famille ont appris qu'elles pourraient être référées à la clinique de gynécologie-oncologie ainsi qu'aux cliniques d'oncologie chirurgicale et de chirurgie plastique en vue de discuter des options en matière de gestion des risques. Malgré la fourniture 
d'informations et d'un soutien d'excellente qualité, et peut-être parce qu'il s'agissait de rendez-vous séparés, les participantes avaient l'impression qu'il leur manquait quelque chose. Des études ont démontré que beaucoup de femmes jugent avoir reçu des informations insuffisantes sur la chirurgie prophylactique.

Selon certaines recherches, quand la prise de décision suit de près la divulgation, il est possible que les femmes éprouvent des niveaux accrus d'angoisse et de détresse, ce qui nuit à leur capacité de retenir des informations et donc à leur capacité décisionnelle.

Rita conseille de se faire accompagner par au moins un membre de la famille (en plus de son époux) afin que cette personne puisse noter l'information et poser les questions que votre époux et vous-même pourriez oublier de poser du fait de votre état d'accablement. J'encourage les infirmières à offrir d'assumer ce rôle lorsque les patients se présentent seuls à ce rendez-vous. Les infirmières et les médecins ne devraient pas supposer qu'une patiente a déjà pris une décision tout simplement parce qu'elle a pris un rendez-vous afin de discuter des options chirurgicales de réduction des risques. La conseillère en génétique et les infirmières en chirurgie et/ou en oncologie ont toutes fourni leur numéro de téléphone et ont encouragé les patientes à les appeler si elles avaient des questions, mais tout le monde n'est pas nécessairement à l'aise avec cette approche privilégiant l'autonomie. Un rendez-vous de suivi avec la conseillère en génétique ou les infirmières en chirurgie afin de passer en revue les options et de répondre aux nouvelles questions favoriserait grandement la prise de décision.

Rita a appris qu'elle devrait prendre un congé d'au moins huit semaines pour sa mastectomie bilatérale avec reconstruction immédiate et un autre de trois à quatre semaines dans le cadre de sa salpingo-ovariectomie bilatérale laparoscopique s'accompagnant d'une hystérectomie vaginale. Elle s'est vue proposer de subir les deux opérations en même temps ce qui réduirait la durée totale de ses congés mais exigerait plus de huit heures d'anesthésie et augmenterait les risques de thrombose veineuse profonde.

Après sa chirurgie des seins, elle allait demeurer à l'hôpital pendant 4 ou 5 jours et recevoir un traitement par ultrasons horaire des seins reconstruits pendant les 24 premières heures. Afin d'éviter de placer un quelconque stress sur son abdomen où les tissus et les vaisseaux sanguins allaient être récoltés, elle sera dans l'impossibilité de se mettre debout bien droite ni de se coucher à plat et devra porter un bandage abdominal à trois panneaux ainsi qu'un soutien-gorge sport 4 semaines durant. Il lui faudra éviter de soulever tout objet faisant plus de $2,5 \mathrm{~kg}$, éviter toute activité sexuelle et tout exercice ardu pendant au moins quatre semaines et ne pas conduire pendant trois semaines.

Ces derniers temps, le public est davantage sensibilisé aux cancers héréditaires par le biais de reportages sur des célébrités dont l'actrice Christina Applegate âgée de 36 ans qui a annoncé, en août 2008, son diagnostic de cancer du sein, le résultat positif de son test de mutation génétique BRCA1 et sa mastectomie bilatérale qu'elle a qualifiée de « décision logique ». Puis, le $1^{\text {er }}$ octobre 2008, la chaîne PBS a diffusé le documentaire «In the Family » de la cinéaste Joanna Rudnick qui, à l'âge de 27 ans, a obtenu un résultat positif au dépistage d'une mutation BRCA.

En dépit des efforts-récents et accrus-de sensibilisation du public, certaines femmes ont signalé que leurs amis et proches étaient « choqués » et « horrifiés » lorsqu'ils prenaient connaissance de leur décision de subir une mastectomie prophylactique, ce qui ne faisait qu'amplifier le stress qu'elles ressentaient et les faisaient hésiter à partager cette information avec quiconque d'autre. J'ai découvert que ce genre de réaction n'est malheureusement pas si rare que cela. Plusieurs infirmières en oncologie, ne sachant pas que ma famille et moi subissions un dépistage génétique, faisaient des commentaires comme les suivants en parlant d'autres patientes : «Je ne sais pas pourquoi une personne peut vouloir subir le dépistage génétiquepuisqu'il n'y a rien qu'elle puisse y faire » et «Comment peuvent- elles songer à subir une chirurgie tellement horrible et mutilante qui, de toute façon, ne leur procurera probablement aucun avantage-si c'était moi, je prendrais mes chances ». Ou l'oncologue qui savait bien que j'attendais les résultats de mes tests génétiques et qui m'a dit : «Berk! Même si ton test est positif, tu ne vas pas subir cette chirurgie-là, hein?»

Avant que la chirurgie prophylactique n'ait lieu, les infirmières doivent aborder avec les patientes la possibilité d'une atteinte à leur image corporelle et l'incidence potentielle de l'opération sur les relations sexuelles des patientes. Il convient de conseiller aux femmes songeant à se prévaloir de ces chirurgies de prendre le temps d'explorer, avec leur partenaire de vie, le sens de la sexualité du couple.

Au départ, Rita avait décidé de subir les opérations en même temps et avait un rendez-vous à cet effet en mars 2007 mais quand la chirurgie du sein a été remise à plus tard, elle a décidé de ne subir que la salpingo-ovariectomie bilatérale de nature prophylactique. Rita avait été prévenue que sa chirurgie du sein pourrait être annulée si l'on avait besoin de son créneau opératoire pour un patient diagnostiqué du cancer. La chirurgie du sein de Rita a ainsi été retardée deux fois, ce qui a redoublé le stress qu'elle éprouvait et l'anxiété de sa famille et lui a causé des problèmes au travail.

À titre d'infirmières en oncologie, nous avons toutes rencontré des patients dont l'employeur ou les collègues pensaient qu'ils profitaient de leurs antécédents médicaux comme autant d'excuses pour obtenir des congés dans le cadre de tests et de rendez-vous de suivi. Ils peuvent laisser entendre que le patient devrait prendre ses rendezvous durant ses jours de congé ou durant ses vacances afin de ne pas causer de nouveaux dérangements à pour leurs collègues et à leurs employeur étant donné que le patient n'est plus vraiment malade et qu'il avait déjà exigé tant de jours de congé maladie quand il recevait son traitement actif. Il y a à parier que les collègues de travail et les employeurs qui embrassent cette notion ne vous offriront guère de soutien lorsque vous aurez besoin de prendre jusqu'à douze jours de congé pour des opérations prophylactiques et que la date de l'une d'entre elles est repoussée deux fois de suite. Et comment diable faisons-nous pour sensibiliser le public quand la réaction première d'oncologues et d'infirmières en oncologie est «Berk! » ou « si c'était moi, je prendrais mes chances! »?

Chez les femmes porteuses de mutations génétiques de BRCA, la prise de décision est une démarche hautement individuelle qui exige qu'elles mettent en balance le risque d'une part, et d'autres priorités dans la vie et des enjeux de qualité de vie, d'autre part. Beaucoup d'entre elles jugent qu'après la chirurgie visant à réduire les risques, elles ont encore besoin de confirmer qu'elles ont fait « le bon choix » en continuant de faire des lectures et des recherches sur les options de réduction des risques et en assistant à des conférences et symposiums s'y rapportant.

\section{Les chirurgies de Rita}

Durant son rendez-vous de septembre 2006, Rita a été avisée par l'équipe de gynécologie-oncologie que son risque de développer un cancer de l'ovaire pouvait atteindre $20 \%$ mais qu'il était probablement moins élevé que ça étant donné qu'elle avait pris, auparavant, des contraceptifs oraux et du tamoxifène. L'équipe lui a également conseillé de songer à subir une hystérectomie puisque certaines données indiquaient qu'elle courait un risque accru de cancer de l'endomètre. Rita, qui a 3 enfants et qui à 58 ans n'était plus en âge de procréer, a trouvé qu'il lui était relativement aisé de prendre la décision concernant ces chirurgies-là. Rita devait subir sa salpingoovariectomie bilatérale prophylactique par laparoscopie et son hystérectomie vaginale, deux opérations avec effraction minimale qui s'accompagnent d'une faible morbidité. On l'a informée qu'elle devrait prendre un congé maladie de trois-quatre semaines. Comme Rita et son mari avaient planifié des vacances en Thaïlande à la fin décembre, il a été décidé, à sa demande, que la chirurgie ait lieu en mars 2007. 
En revanche, la décision concernant la prise en charge de son risque de cancer du sein a exigé davantage de considération et de délibération. Au début, Rita semblait pencher pour la surveillance accrue. À son rendez-vous d'octobre 2006, la chirurgienne-oncologue a passé en revue les options possibles. À son avis, la surveillance ne constituait pas une option valable dans le cas de Rita puisqu'elle revient tout simplement à espérer pouvoir détecter le cancer du sein à un stade précoce. La chimioprévention ne constituait pas non plus une option valable puisque Rita avait déjà suivi un traitement au tamoxifène. Elle ajoutait qu'il lui était difficile de donner une estimation précise du risque qu'encourait Rita de développer un autre cancer du sein mais elle le situait à au moins $25 \%$, probablement plus. Elle recommandait une mastectomie bilatérale prophylactique avec ou sans reconstruction. Elle a assuré à Rita qu'elle n'avait pas besoin de prendre une décision immédiate et lui a recommandé de rencontrer un chirurgien plastique afin de discuter des options en matière de reconstruction. En attendant que Rita prenne sa décision, elle a commandé une IRM des seins et lui a réservé un rendez-vous de suivi dans six mois.

Rita a rencontré le chirurgien plastique en novembre. Il a passé en revue les risques, les avantages et les différentes options de reconstruction faisant appel à des tissus autogènes, a décrit la technique du lambeau perforant de l'artère épigastrique inférieure aussi appelée technique du lambeau DIEP ainsi que l'anastomose microvasculaire. Cette méthode de reconstruction permet d'utiliser les vaisseaux sanguins du muscle ainsi que le tissu adipeux et l'épiderme. Comme le muscle est gardé intact, la force des abdominaux n'est pas compromise et la douleur est moindre. En prime, la femme a ainsi droit à une abdominoplastie en plus de la reconstruction. La reconstruction immédiate a lieu tandis que la patiente est encore sur la table d'opération à la suite de sa mastectomie, ce qui permet de réduire la durée totale du séjour à l'hôpital et de l'arrêt de travail. Selon les estimations, seulement $20 \%$ des Nord-Américaines ont accès à la technique du lambeau DIEP, nous avons donc beaucoup de chance que cette opération soit communément offerte au Manitoba. Afin de rehausser le résultat esthétique, la chirurgienne-oncologue réalisant la mastectomie utilise une incision similaire à celle utilisée dans la réduction mammaire; elle a expliqué à Rita qu'elle s'en servirait sur le sein droit et éventuellement sur le sein gauche, mais qu'elle s'inquiétait de la viabilité de l'épiderme en cet endroit du fait de sa chirurgie et de sa radiothérapie antérieures. À cause de cela, Rita pourrait avoir besoin de subir une nouvelle opération quelques mois plus tard afin de « peaufiner » le résultat esthétique. Après la guérison, les mamelons sont créés et les auréoles tatouées.

L'une des recommandations de Rita est que les infirmières en chirurgie doivent montrer à chaque patiente, avant son congé de l'hôpital, de quoi a l'air le champ opératoire et l'évolution prévue. Il serait également utile d'aborder les diverses complications hypothétiques - que faire si un des drains se détachait, que faire si l'une des sutures se défaisait, etc. Cet enseignement devrait inclure l'époux de la patiente et être offert à leurs enfants adultes si la patiente le souhaite. Les infirmières doivent avoir conscience des problèmes éventuels en matière d'image corporelle et de sexualité et de la détresse associée durant les douze premiers mois suivant la chirurgie prophylactique. Des consultations de soutien doivent être offertes aux patients et à leurs proches avant la chirurgie et jusqu'à un an après.

\section{L'annonce aux enfants}

Comme je l'ai mentionné ci-haut à propos du dépistage des mutations BRCA, il est généralement recommandé d'attendre que l'individu ait atteint l'âge où il peut prendre des décisions éclairées à son sujet. Selon l'avis majoritaire, «l'âge approprié » pour se livrer au dépistage est 25 ans ou 5-10 années avant le plus précoce diagnostic de cancer du sein jamais posé dans la famille.
Une collègue infirmière dont une amie est porteuse d'une mutation génétique BRCA1 m'a confié : «Il est si ardu de savoir quand il est opportun d'apprendre la nouvelle aux enfants ».

Quoiqu'il existe peu d'information sur le moment opportun et sur le comment de la divulgation de l'information aux enfants, les membres de ma famille ont des opinions bien arrêtées et divergentes à ce sujet.

D'après Rita et ses proches, il est important de ne rien cacher à ses petits-enfants. «Il faut qu'ils voient ce que je fais pour éviter toute nouvelle apparition du cancer, l'excellente façon dont je m'adapte et le bonheur que je ressens. »

L'une de nos nièces ne transige pas : ses enfants ne doivent pas l'apprendre tant qu'ils ne sont pas assez âgés pour prendre leurs propres décisions. Elle estime que ses enfants ont traversé suffisamment d'épreuves après avoir appris que leur mère avait le cancer du sein et l'avoir vue faire face à ses chirurgies, sa chimiothérapie et sa radiothérapie.

Sa sœur, qui est également porteuse de la mutation génétique BRCA2 mais n'a jamais eu le cancer, a informé ses enfants de sa situation en leur donnant des informations adaptées à leur âge et leur a expliqué, de façon toute naturelle, pourquoi elle avait opté pour la chirurgie prophylactique. Elle ajoutait qu'il lui paraissait injuste de priver ses enfants de cette information puisqu'il « s'agit d'un événement de la vie familiale ». N'empêche, m'a-t-elle confié, qu'elle s'inquiète du fait que ce savoir pourrait déboucher sur la stigmatisation. Cela allait-il créer des problèmes au niveau des relations que les enfants établiraient-il se peut que certaines personnes ne veuillent pas prendre le risque d'épouser ou d'embaucher quelqu'un dont les résultats de dépistage pourraient être positifs. Elle se tracassait aussi de la possibilité qu'un de ses enfants en parle à ses cousins et aggrave ainsi la détresse de sa sœur.

Il est donc capital que l'on effectue plus de recherches et que l'on dispose de davantage d'information sur l'impact de cette divulgation sur les enfants. La recherche infirmière n'a-t-elle pas là une excellente opportunité de combler la lacune.

\section{Assimiler le savoir- et même le transcender}

On dit souvent que le cancer change la vie de bien des manières et qu'il la change à jamais.

Presque tous les survivants du cancer seront d'accord avec moi pour affirmer qu'ils ont désormais, grâce à l'apparition d'un cancer, une plus vive appréciation de la vie mais je n'ai jamais rencontré qui que ce soit qui avait le cancer et qui ne s'inquiétait pas à un degré ou à un autre de la possibilité d'une récidive, notamment lorsque la personne souffrait d'une autre maladie ou juste avant les rendez-vous de suivi réguliers.

Au printemps 2008, Rita a attrapé un rhume dont elle ne semblait pas pouvoir se débarrasser lequel a abouti, quelques semaines plus tard, à l'apparition d'une toux sèche et grave, particulièrement pénible la nuit, s'accompagnant d'un essoufflement à l'effort, d'une fatigue et d'une lassitude considérables. Elle s'est forcée à continuer de travailler à temps plein et à travailler sur demande puisqu'une de ses collègues avait besoin de prendre des congés pour aider son père malade. Au bout de quelques semaines, Rita a commencé à éprouver, à chaque nouvel accès de toux, une douleur aiguë sur le côté inférieur de la cage thoracique et elle a pensé que sa toux persistante avait fini par lui fracturer une côte. Elle en a parlé à un des médecins de l'hôpital où elle travaille et ce dernier a commandé une radiographie laquelle a révélé un épanchement pleural du côté droit. Ce même médecin a fourni à Rita une ordonnance d'antibiotique et conscient des antécédents de Rita en matière de cancer du sein, lui a fait subir d'urgence une tomographie par ordinateur de la poitrine qui n'a révélé aucune autre anomalie mise à part l'épanchement pleural. Rita a été dirigée vers la médecine thoracique à des fins d'évaluation et de 
thoracentèse diagnostique. La cytologie n'a permis de déceler aucune cellule maligne. Il a été déterminé que son épanchement était exsudatif, qu'il s'agissait d'une inflammation provoquée par une pneumonie. Quoique nous sachions tous que son épanchement pleural était probablement dénué de toute malignité - après tout, ses tissus ne présentaient aucun signe de malignité après ses mastectomies-la crainte est toujours présente, à divers degrés.

L'approche de vie de Rita est de «prendre les choses comme elles viennent » et pour elle, «l'inquiétude est une perte de temps et d'imagination ». Comme elle profite au maximum de chaque journée et jouit réellement de la vie, elle constitue un excellent modèle pour tout le monde. L'un des trois enfants de Rita a également obtenu un résultat positif au dépistage de la mutation génétique BRCA2. Avant d'apprendre les résultats de ses tests, ce dernier a déclaré qu'il était inutile de se tracasser de quelque chose qui était déjà déterminé. Il croyait qu'il était prêt à recevoir un résultat positif mais à l'annonce de la mauvaise nouvelle, il a été surpris à quel point il en était bouleversé au départ. Car il n'était pas le seul à être touché par cette nouvelle - elle pouvait avoir une incidence sur la santé et le bien-être de ses enfants, et il allait devoir vivre dans l'incertitude jusqu'à ce qu'ils parviennent à l'âge adulte. Ayant toujours ce savoir à l'esprit, nous avons conscience que lorsque la prochaine génération atteindra l'âge adulte et sera amenée à prendre ses décisions sur le dépistage génétique, nous prierons de nouveau que la chance sur deux soit de notre côté.

Je suis consciente qu'il y a des gens, dans d'autres familles, qui ont décliné le dépistage génétique parce qu'ils «se sentent mieux de ne pas savoir » et d'autres qui ont obtenu des résultats positifs et qui se sont tourmentés pendant des mois et des mois à propos des options se présentant à eux. Pourtant, il y en a d'autres, beaucoup d'autres, qui pensent que le fait de savoir les a habilités à prendre connaissance de leur risque de cancer et des options permettant de réduire ce risque. Tandis que j'attendais mes résultats au test génétique, une grande amie qui était atteinte d'un cancer du sein métastatique depuis un peu plus de cinq ans m'a dit : «Si ton résultat est positif, n’hésite pas. Fais tout ce que tu peux afin de réduire tes risques. Je donnerais tout ce que je possède pour avoir eu cette opportunité-là. La vie est tellement précieuse! »

Ne nous laissons pas aveugler par les horloges et les calendriers; chaque moment de la vie est à la fois un miracle et un mystère. -H.G. Wells [traduction libre]

\section{Conclusion}

L'impact de la prise de connaissance des résultats aux tests de prédisposition génétique peut durer toute la vie et même plus. Les infirmières doivent donc se pencher sur les interactions qu'elles entretiennent avec les femmes ayant obtenu un résultat positif ou non concluant au test de dépistage de la mutation BRCA, discutent de l'information relative au risque avec les patients et leurs proches, fournissent des options de suivi après la réalisation du dépistage génétique et enfin, offrent une planification à long terme en matière de réduction des risques. Les femmes songeant à subir une chirurgie prophylactique ont besoin d'avoir en face d'elles des interlocuteurs bienveillants et bien informés et de trouver un endroit sûr où elles peuvent vraiment exprimer leurs sentiments.

Voilà 16 ans que Helene Hudson est décédée prématurément et 17 ans que ma sœur Rita a été diagnostiquée du cancer du sein. Durant ces années-là, nous avons acquis les connaissances et les compétences permettant d'identifier avec une meilleure précision les personnes à risque de développer le cancer du sein et d'offrir des options de réduction des risques. Pour les infirmières, se tenir suffisamment informées pour pouvoir incorporer les informations génétiques les plus actuelles dans leur enseignement et leur appui aux patients est un défi de taille, mais en tant que membres clés des équipes de santé, c'est un défi que nous nous devons de relever. Je sais que Helene Hudson le ferait si elle était encore parmi nous.

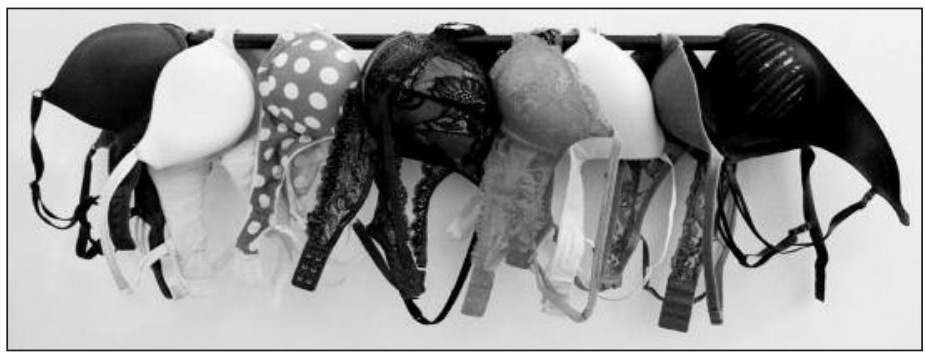

\section{Remerciements}

$D^{r}$ Edward Buchel, directeur, Chirurgie plastique, Université du Manitoba; chef, Chirurgie, Centre des sciences de la santé, Winnipeg, et $D^{r}$ Tom Hayakawa, Chirurgie plastique, Centre des sciences de la santé, Winnipeg et Action Cancer Manitoba.

\section{Références}

Fackenthal, J.D., \& Olopade, O.I. (2007). Breast cancer risk associated with BRCA 1 and BRCA 2 in diverse populations. Nat Rev Cancer, 7(12) 937-948.

Fatouras, M., et al. (2007). The predominant role of surgery in the prevention and new trends in the surgical treatment of women with BRCA 1/2 mutations. Annals of Surgical Oncology, 15(1), 21-33.

Greco, K.E., \& Mahon, S. (2004). Common hereditary cancer syndromes. Seminars in Oncology Nursing, 20(3), 164-177.

Horsman, D., et al. (2007). Clinical management recommendations for surveillance and risk-reduction strategies for hereditary breast and ovarian cancer among individuals carrying a deleterious BRCA1 or BRCA2 mutation. Women's Health JOGC, 45-60.

Kenen, R.H., et al. (2007). Women with BRCA 1 or BRCA 2 mutations renegotiating a post-prophylactic mastectomy identity: Self-image and self-disclosure. Journal of Genetic Counselling, 16, 789-798.

Lodder, L.N., et al. (2002). One year follow-up of women opting for presymptomatic testing for BRCA 1 and BRCA 2: Emotional impact of the test outcome and decisions on risk management (surveillance or prophylactic surgery). Breast Cancer Research and Treatment, 73, 97-112.

National Cancer Institute, www.cancer.gov/cancertopics/factsheet/ risk/BRCA

National Comprehensive Cancer Network. (2008). NCCN clinical practice guidelines in oncology: Genetic/familial high-risk assessment: Breast and ovarian V.1. Fort Washington, PA: Author.

Peshkin, B.N., \& Issacs, C. (n.d.). Risk assessment in women with an inherited predisposition to breast cancer. Retrieved from www.utdol.com

Rodriquez, E., \& Domochek, S.M. (2007). The prevention of hereditary breast cancer. Seminars in Oncology, 401-405.

Société canadienne du cancer, 2007. www.cancer.ca/canada-wide.aspx van Dijik, S., et al. (2008). Decision making regarding prophylactic mastectomy: Stability of preferences and the impact of anticipated feeling of regret. Journal of Clinical Oncology, 26(14), 2358-2363.

Wiley, S.C., \& Cocilova, C. (2007). Screening and follow-up of the patient at high risk for breast cancer. Obstetrics \& Gynecology, 110(6), 1404-1416. 